Original Research Paper

\title{
Application of a Multi Stage Method to Assess the Landscape Alteration Induced by Quarrying Sites: A Comparative Analysis
}

\author{
Dario Lippiello, Guido Alfaro Degan, Marco De Agostini and Mario Pinzari \\ Department of Engineering, University of Roma Tre, Rome, Italy
}

\author{
Article history \\ Received: 27-05-2016 \\ Revised: 01-09-2016 \\ Accepted: 02-09-2016 \\ Corresponding Author: \\ Dario Lippiello \\ Engineering Department, \\ University of Roma Tre, Rome, \\ Italy \\ Email: dario.lippiello@uniroma3.it
}

\section{Introduction}

Due to increasing environmental awareness, nowadays both the public sphere and state administrators pay particular attention to the impact any industrial site has on its surrounding environment. The extractive industries are considered among the most sensitive sectors in this regard as they have substantial environmental externalities, not least of which being their visual impact on the landscape. Whilst the environmental impact of physical and chemical agents may be assessed objectively using on-site measurements, the visual impact of extractive activities is also linked to observers' perceptions and is consequently more subjective. Moreover, the typical pollutant agents produced by a quarry may be controlled through corrective procedures whose effects are immediately appreciable, whereas any changes to the landscape as a result of quarrying are much more difficult to rectify and are generally long-lasting. This seems to suggest that in order to minimize the visual impact of a quarry, a preventative approach would be required during the design stage. The assessment of visual impact may be divided into two different phases (Pinzari and Valente, 1992): Firstly the evaluation of the physical alteration of the landscape and secondly an evaluation of the perception of this alteration from an observer's perspective. Assessment of the visual qualities of landscape alteration has been dealt with by many authors using different approaches (Shafer, 1969; Briggs and France, 1980), taking into account subjective perception (Massacci and Dentoni, 2007; Mouflis et al., 2008; Panagopoulos et al., 2007) or a hierarchy of various landscape components (Kumar Dey and Ramcharan, 2008). More recent studies (Bishop, 2003) based on the recourse to photographs (Pinto et al., 2002) and to 3D models allowed a huge set of data to be collected to validate this approach. On the other hand, the assessment of the physical alteration of a site assumes a certain 
importance during the planning stage (Menegaki and Kaliampakos, 2006) before work commences at the site and some constraints are determined such as receptors to be screened or sensitive areas to be preserved (Alfaro Degan et al., 2014). During this phase the designer plays a crucial role by deciding upon of the extraction method (Cardu et al., 2001) and the reclamation plan (Gasparovic et al., 2009; Jordan, 2007). Any existing or future urbanization in the surrounding area may also represent a significant parameter (Hernandez et al., 2004). The aim of this study is to define a simple procedure based on a limited number of parameters to assess the visual impact of extractive activities. Such an approach may be used as a tool to compare different extraction methods or reclamation plans and, used in conjunction with other planning techniques during the design stage, to quantitatively forecast the alteration to the landscape due to expanding the quarried surface or the effectiveness of reclamation plans. The procedure is tested for validity on two case studies in Italy.

\section{Materials and Methods}

The assessment procedure is divided into three logical steps, each of which is characterized by a quantitative parameter. This structure allows the designer not only to upgrade the analysis, but also to discontinue it should a rapid overview prove satisfactory.

The first step aims to define the characteristics of the quarry. Recent studies (Menegaki et al., 2015), have underlined the fact that observers tend to take particular notice of the chromatic contrast between the excavated area and the colour around the edge of the site and this aspect of alteration tends to vary according to the characteristics of the material being quarried. Hence, one of the first parameters to be quantified is the extent of the Exposed Quarried Area (EQA) which varies considerably according to the position of the receptor (i.e., the viewpoint) and the choice of the exploitation method. The EQA is considered as being the projection of the visible area of the quarry onto the plane view which is identified according to the choice of the receptor position. The view plane depends on the angle that the direction of vision (the line traced from the receptor to the quarry) forms with the horizontal plane. The view plane is orthogonal to the direction of vision (as shown in Fig. 1). When many viewpoints around the site are included, the EQA also depends on the azimuthal angle allowing an omnidirectional analysis to be made. Thus, in order to provide a more detailed appraisal, various viewpoints were considered from all around the sites in these case studies and polar diagrams were drawn up to represent the extent of the EQA. Moreover, the evolution of this parameter was also monitored throughout the quarry's lifetime to provide a comparative analysis of the results.

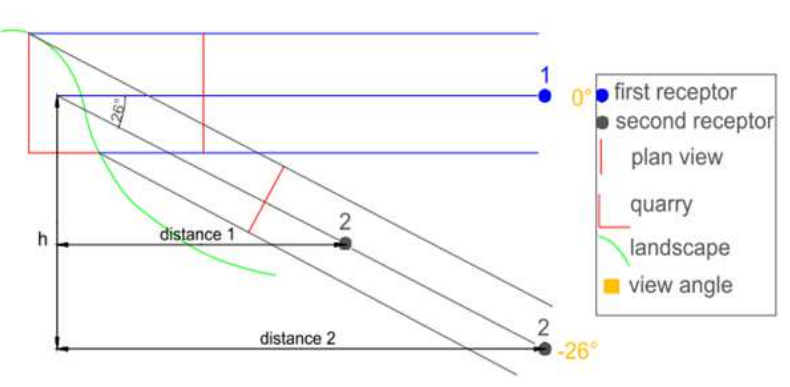

Fig. 1. Exposed quarry area and corresponding parameters

The second step defines from which areas of the surrounding land the quarried surface is visible. This step requires a graphical analysis of the topography of the surrounding area and may be assessed by utilising the extent of the Zone of Visual Influence (ZVI) as the quantitative parameter. In order to assess the ZVI, firstly a cone of visibility is plotted, which is obtained graphically from the polar diagram and is constituted by the azimuthal angle within which the quarry is visible. In particular it is drawn up as follows: The apex is represented by the quarry site that is the center of the polar diagram itself. The projections of the generatrices onto the horizontal plane are obtained by tracing the tangents from the center of the diagram to the circumferences representing the EQA previously assessed (Fig. 14 and 15). Then the surrounding topography is analysed to verify whether the quarry is in fact visible from the whole area within the cone of visibility. The visibility chart representing the ZVI is plotted considering, for any given receptor around the quarry, angle $\gamma_{i}$ and the corresponding orography. The angle $\gamma_{i}$ is calculated with the following Equation 1:

$\gamma_{i}=\operatorname{arcos}\left(\frac{B}{A}\right)$

whose parameters are represented in Fig. 2 in which A is the distance connecting the observation point to the highest point of the quarry and $\mathrm{B}$ is its projection onto the horizontal plane.

Defining the critical angle $\gamma_{c i}$ as the quarry's slope angle in the particular case when A connects the highest and the lowest points of the quarry, for each direction $i$, the quarried surface is visible when $\gamma_{i}$ is smaller than $\gamma_{c i}$ as shown in Fig. 3.

This allows the visual impact of the quarry on its surroundings to be quantified in terms of the extent of the area from which it may be seen by potential observers. This step was carried out for the two selected sites according to maps which were plotted to correspond to various stages of the exploitation of the quarry (after 1, 3, 5 and 10 years respectively). 


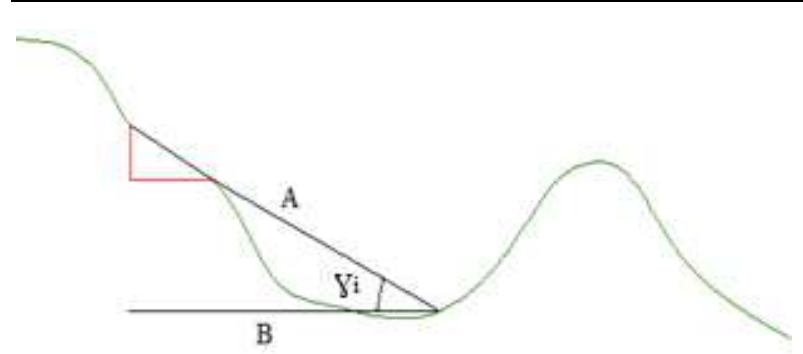

Fig. 2. Angle of vision

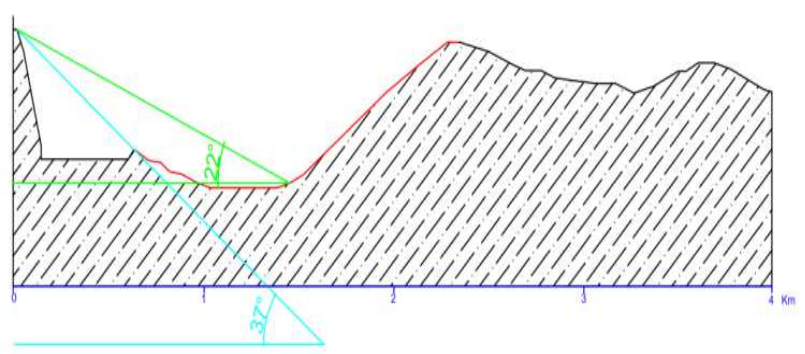

Fig. 3. View angle (green) and critical angle (blue)

The third and final step is intended to consider the combined effects of the design choices and the effects on the surrounding site. This step, performed by adopting the mean critical angle and the extent of the ZVI, is fundamental as it allows a design parameter to be set whose calibration helps to minimize the two previous ones. As described above, the critical angle is the maximum value above which the excavated area is no longer visible to the observer. This value also depends on the quarrying method adopted (the project choice) and more specifically on the amount of excavation that has taken place until the point in time in question. Within the cone of visibility and its corresponding azimuthal angle of visibility, a number of visual directions $\mathrm{N}$, appropriate to the size of the quarry, were drawn from the centroid of the quarry outwards through the visibility zone. The critical angle was determined for each direction and the mean value was then calculated as follows:

$$
\gamma_{c, m}=\sqrt[3]{\frac{\sum_{i=1}^{N} \gamma_{c, 1}^{3}}{N}}
$$

Where:

$N=$ Represents the number of sections considered within the cone of visibility.

$\gamma_{c i}=$ Represents the value of the critical angle in the direction identified in the $\mathrm{i}$-th section.

$\gamma_{c m}=$ Represents the value of the mean critical angle within the cone of visibility.

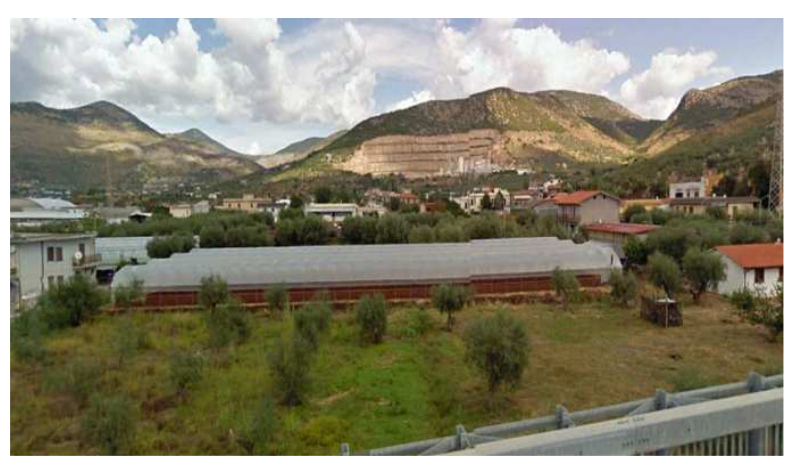

Fig. 4. Quarry at site A partially excavated

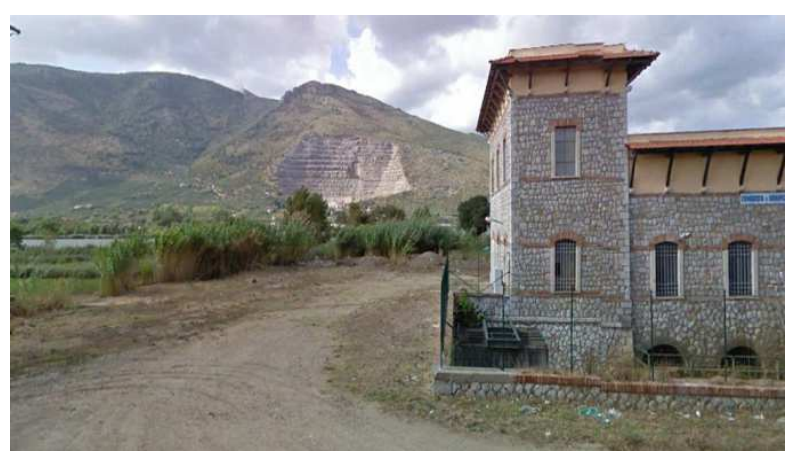

Fig. 5. Quarry at site B partially excavated

The adoption of this parameter together with the extent of the ZVI allows the Inter Visibility Diagram (IVD) to be defined, visually indicating the effect that the choice of quarrying method has on the size of the ZVI. The minimum level of the IVD allows design parameters to be set, thus minimizing the corresponding visual impact.

The sites of the two case studies are both limestone quarries located near Terracina in the Lazio region of Italy and have both been partially excavated as seen in Fig. 4 and 5.

Although the two quarry sites have similar terrain topography, their dimensions are different. The first quarry, at site A, overlooks Terracina and is less than 4 $\mathrm{km}$ from coastline. It is located on the southeast side of a hill whose summit is approximately $260 \mathrm{~m}$ above sea level. The second quarry, site B, overlooks Lake Fondi and is less than $5 \mathrm{~km}$ from the coast on the southwest side of a $300 \mathrm{~m}$ high hill. The surrounding area near the coast is almost flat and has an average altitude of $30 \mathrm{~m}$ above sea level. The area of the quarry at site A is 8.5 hectares, while that of the quarry at site B is 11.8 hectares.

The analysis was carried out by examining not only the exploitation method adopted in reality, but also other alternative exploitation methods. The selection of exploitation methods for the comparative analysis was performed by taking into account both the dimensional and mechanical characteristics of the two deposits. The only three methods that revealed to be compatible with the 
characteristics of both deposits were single bench, vertical slices and single bench with reclamation. So both sites were plotted as they would be quarried by each of three different methods: Single bench, vertical slices and single bench with reclamation. In both single bench methods the material is extracted from a single level or bench; the excavation work then proceeds to the level below after work on the first level has been completed (a top down or descending method). These two options differ from each other according to whether the reclamation is carried out on completion of each bench, or if reclamation is left until the end of the quarry's lifetime. On the other hand, the vertical slices method consists in removing material by cutting sub vertically in single elements from the top of the quarry to the quarry floor. In Fig. 6 and 7 the scheduling of how the mineral deposit is to be subdivided and quarried is shown both for single bench and vertical slices method.

Assuming the yearly production as an input data fixed at $440000 \mathrm{~m}^{3}$, each configuration was plotted accordingly. Under this assumption, the only difference between the two sites with regard to production is the total duration of the quarrying activity. According to estimates of the deposits at the two sites, the total time required from start to finish to completely excavate the deposit at site $\mathrm{A}$ is about five years, while the total quarrying time required for site $\mathrm{B}$ is about ten years. Three stages of quarry development were plotted (referring to the beginning, the middle and the end of the lifetime of the quarry in question); thus the layouts regarding the quarry at site $\mathrm{A}$ refer to the first, third and fifth years of its lifetime and those regarding the quarry at site B show the first, fifth and tenth years of its lifetime, respectively. Each figure has contour lines indicating an altitude interval of five meters between each line.

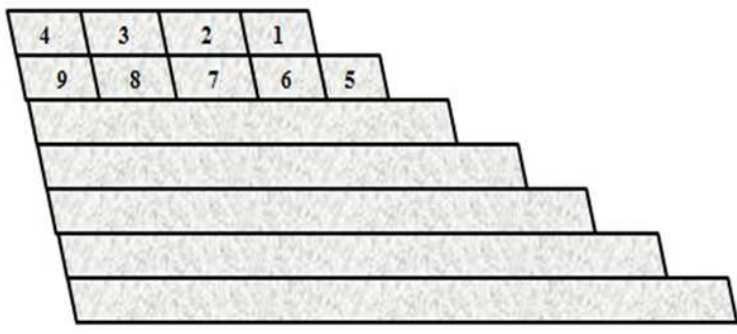

Fig. 6. Single bench method

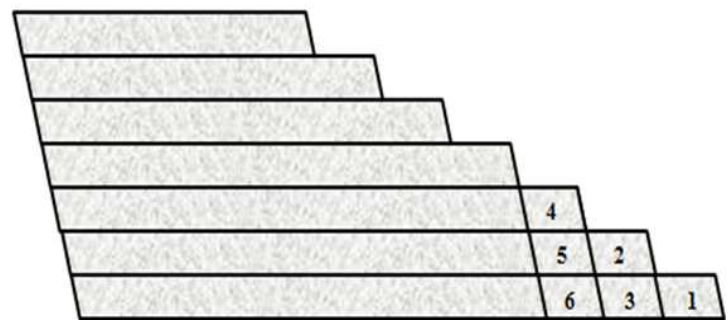

Fig. 7. Vertical slices method
In Fig. 8-10 some examples are shown regarding the final layouts (fifth year) at site A, while in Fig. 11-13 final layouts (tenth year) regarding site B are shown.

The final step is aimed to test the validity of the proposed method by checking its accuracy. Data obtained by means of the designed procedure are put in comparison with those measured in reality for both sites.

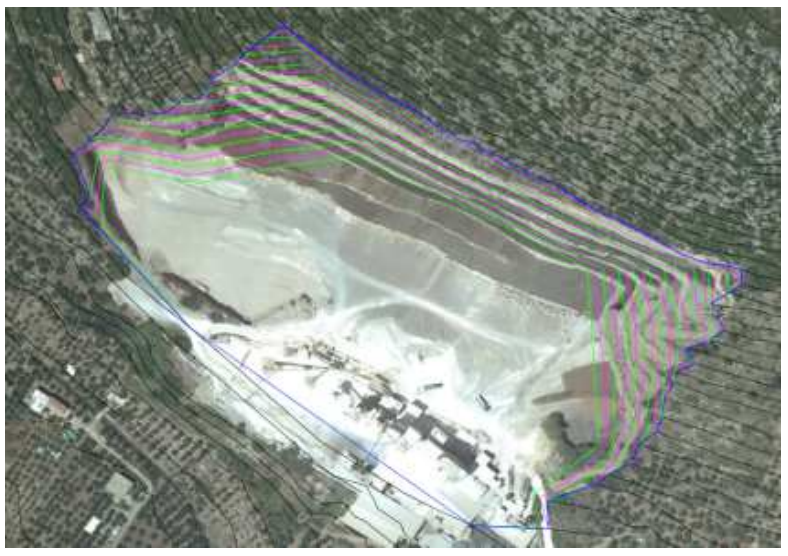

Fig. 8. Single bench method after 5 years

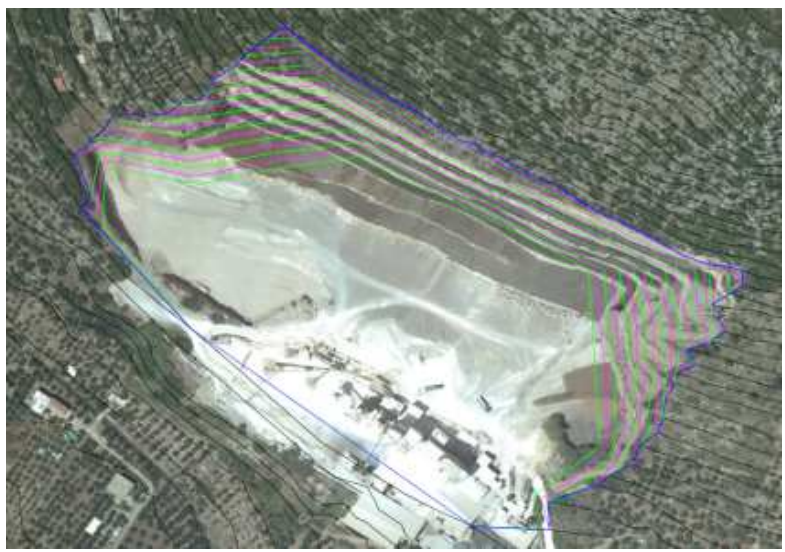

Fig. 9. Vertical slices method after 5 years

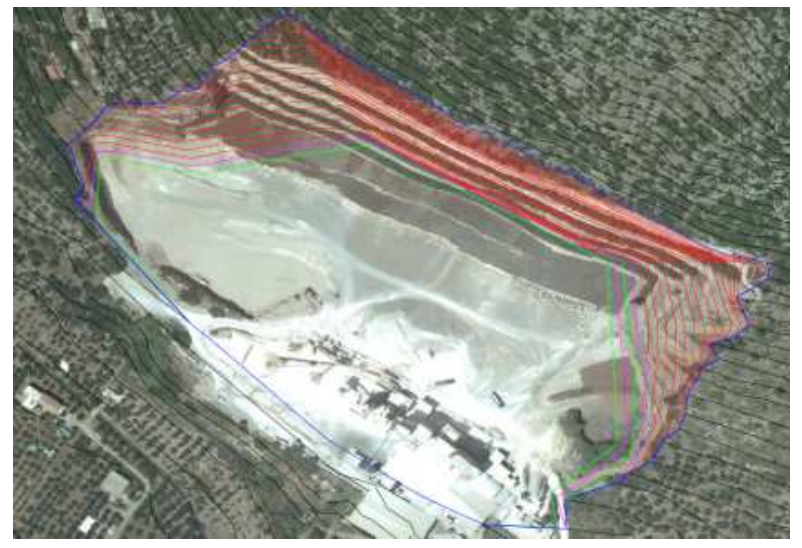

Fig. 10. Single bench with reclamation method (red lines represent the restored part of the quarry) after 5 years 


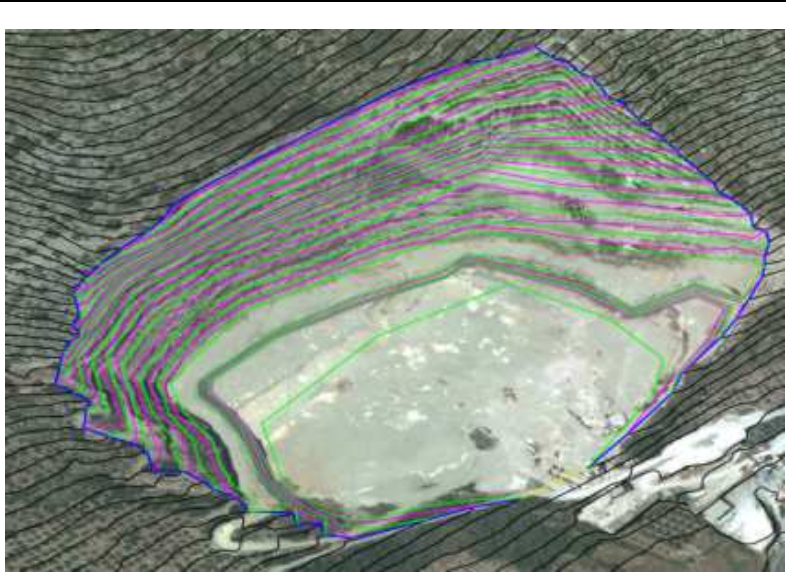

Fig. 11. Single bench method after 10 years

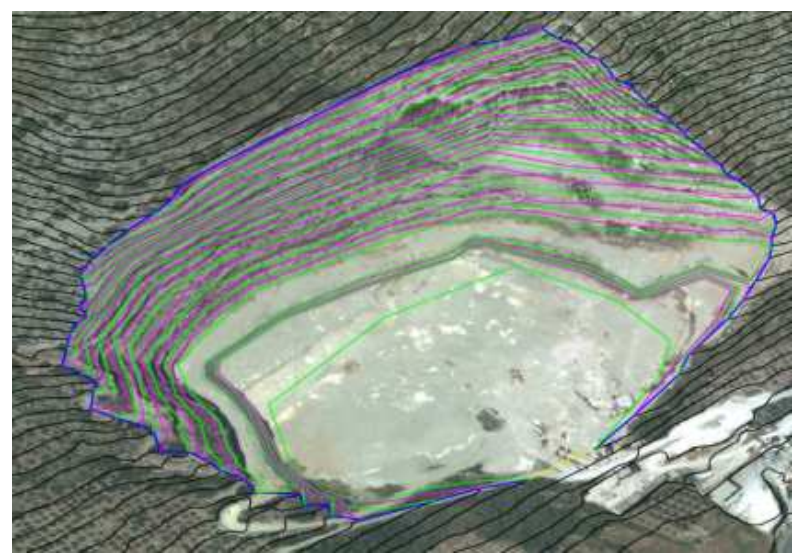

Fig. 12. Vertical slices method after 10 years

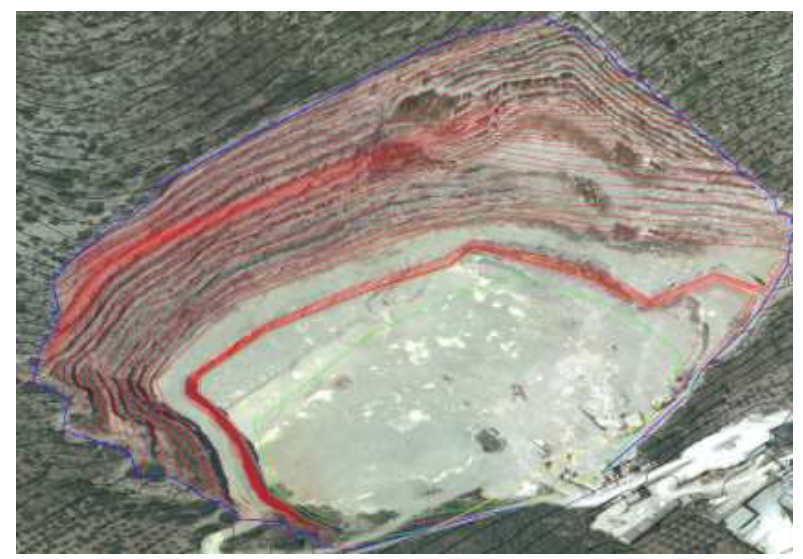

Fig. 13. Single bench with reclamation method after 10 years (red lines represent the restored part of the quarry)

\section{Results}

The results obtained from analysing the three layouts are summarized in the following three steps. The first one is constituted by the assessment of EQA. The maximum values of EQA for each site at the various time intervals are provided in Table 1 and 2.

\begin{tabular}{llll}
\multicolumn{3}{l}{ Table 1. Exposed quarry area at site A } \\
\hline & Max exposed quarry area $\left[\mathrm{m}^{2}\right]$ \\
& $-\mathrm{y}-\mathrm{-ar}$ 1 & Year 3 & Year 5 \\
Exploitation method & Yea--- \\
\hline Single bench & $16.869,38$ & $30.073,00$ & $38.124,35$ \\
Vertical slices & $11.354,44$ & $23.973,00$ & $38.124,35$ \\
Single bench with recl. & $16.869,38$ & $14.686,93$ & $4.348,89$ \\
\hline
\end{tabular}

Table 2. Exposed quarry area at site B

\begin{tabular}{|c|c|c|c|}
\hline \multirow[b]{2}{*}{ Exploitation method } & \multicolumn{3}{|c|}{ Max exposed quarry area $\left[\mathrm{m}^{2}\right]$} \\
\hline & Year 1 & Year 5 & Year 10 \\
\hline Single bench & $22.206,38$ & $49.489,99$ & $66.832,83$ \\
\hline Vertical slices & $10.553,18$ & $35.498,307$ & $66.832,83$ \\
\hline Single bench with recl & $22.206,30$ & $10.472,06$ & $5.369,87$ \\
\hline
\end{tabular}

The parameter of maximum EQA allows the extent of the landscape alteration due to quarried surface to be quantified quickly, without regard to the view direction.

The Exposed Quarry Area (EQA) is indicated in a more compact way by means of polar diagrams. The different colours represent the progress of excavation throughout the quarry's lifetime: Green (beginning of excavation), yellow (middle of excavation), blue (end of excavation). In order to facilitate the interpretation of the polar diagrams the angle of $0^{\circ}$ corresponds to magnetic North. The extent of EQA is expressed as $10^{3} \mathrm{~m}^{2}$. Finally for each of the figures, the view angle $\gamma_{\mathrm{i}}$ is set to $0^{\circ}$ supposing the viewer is standing at the same height above sea level as the foot of the quarry.

Once the polar diagram has been drawn, plotting the tangents from centre of the diagram to the circumferences obtained previously helps to define the cone of visibility itself. This step allows an angle (of visibility) to be identified within which the topography must be analysed in order to plot the ZVI. This step is shown for site A in Fig. 16. The visibility chart, in this case, is obtained by considering 18 sections with an angle of $8^{\circ}$ degrees between each section resulting in a total angle of visibility of $144^{\circ}$.

This procedure, together with the comparison between the view angle and the critical angle for each section, allows maps of the ZVI to be plotted. The Fig. 17 shows the visibility chart when the single bench with reclamation method is performed at site $\mathrm{A}$ and illustrates the changes in the ZVI as the excavation progresses over the years according to the exploitation method adopted. The area marked in violet refers to the first year, green to the third, yellow to the fifth.

With regard to the visibility charts of site $\mathrm{B}$, the ZVI is provided for the first year (marked in violet), the fifth year (green) and the tenth year (yellow). In the Fig. 18, the visibility chart is provided when single bench with reclamation method is performed.

The extent of each area was assessed and their corresponding values are reported in Table 3 and 4 . 

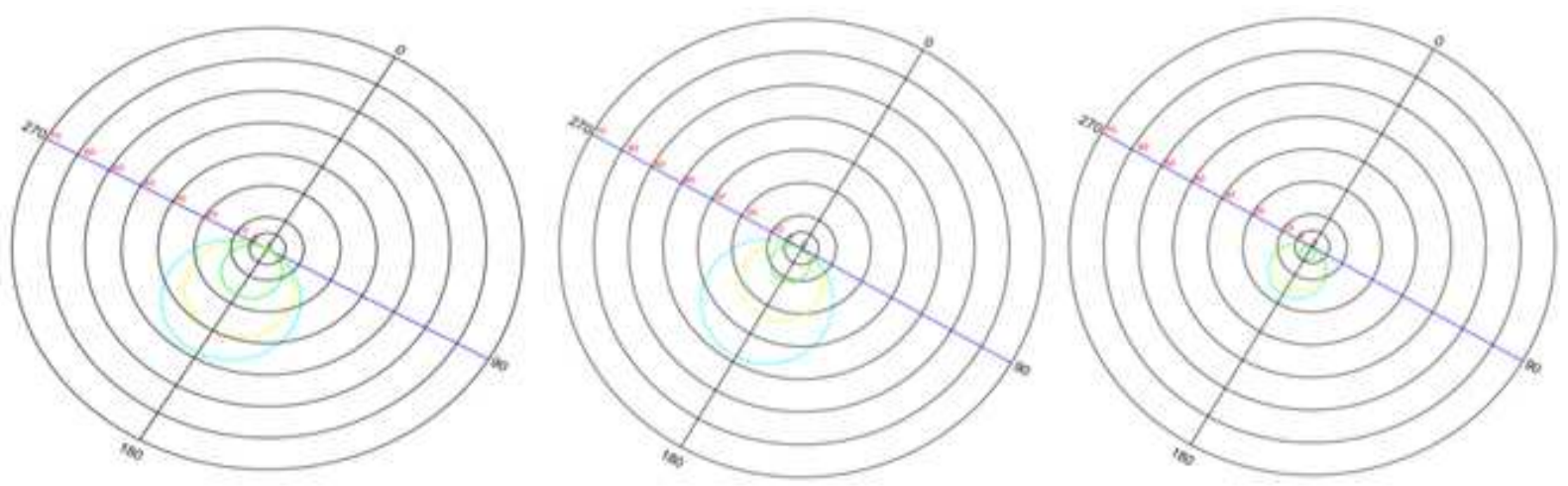

Fig. 14. Site A: Polar diagrams representing EQA (single bench on the left, vertical slices in the middle, single bench with reclamation on the right)
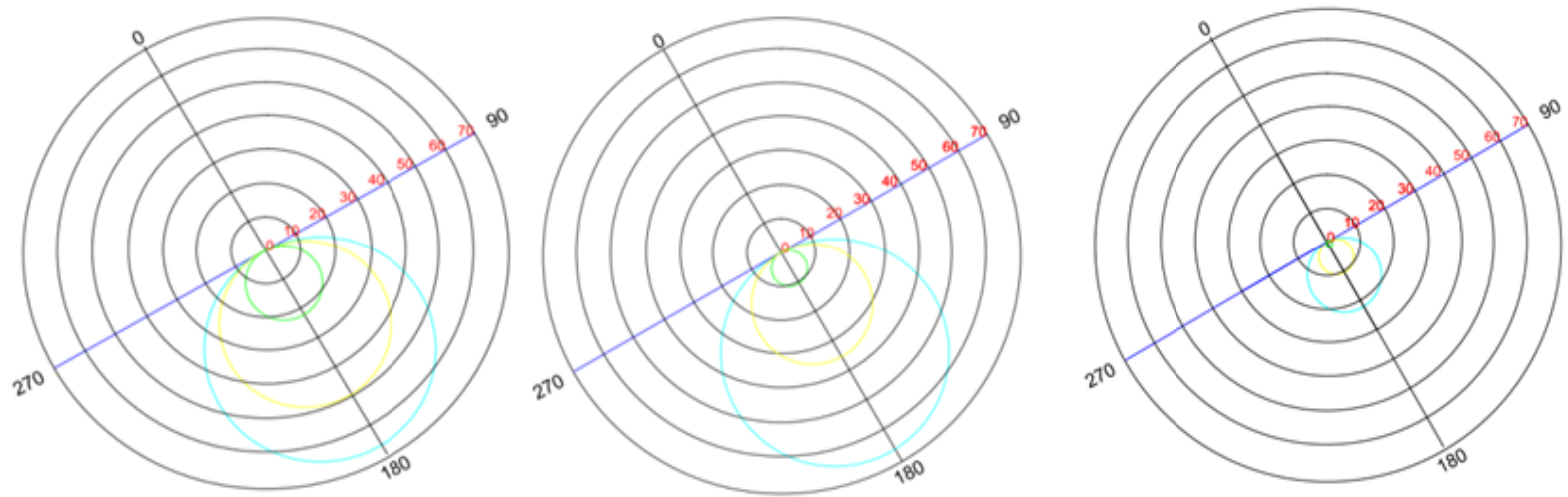

Fig. 15. Site B: polar diagrams representing EQA (single bench on the left, vertical slices in the middle, single bench with reclamation on the right)

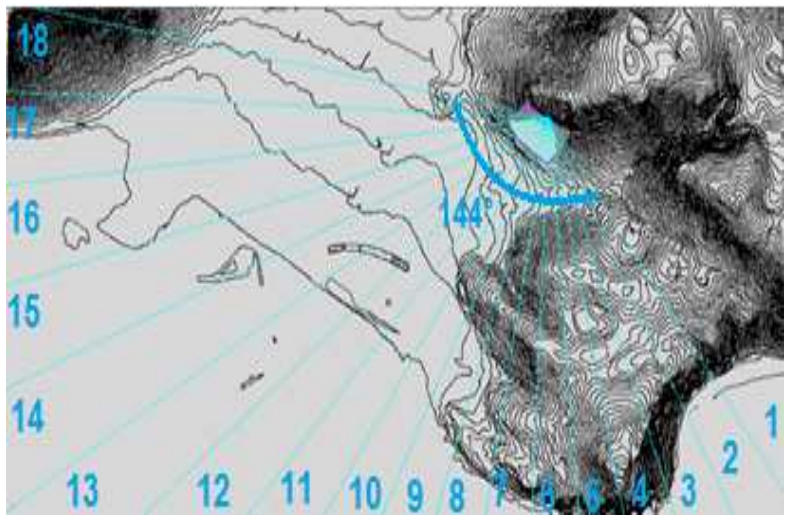

Fig. 16. Site A, 18 sections used to construct the visibility charts

The objective of the third and final step is to merge the parameter of the critical angle with that of the extent of the ZVI. To this aim the value of the critical angle is assessed for each of the 18 sections within the angle of visibility and the mean value is then calculated according to Equation 2. The values obtained are expressed in degrees and are summarized in Table 5 and 6 according to the three methods of excavation over the respective quarry's lifetime.

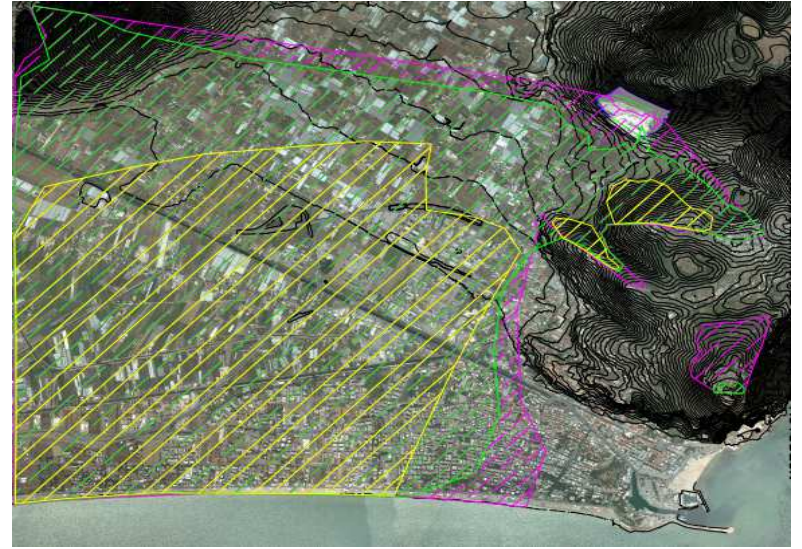

Fig. 17. Site A, Single bench with reclamation visibility chart

The final correlation is set according to the Inter Visibility Diagram in which the extent of the ZVI is compared with the mean critical angle. The IVD diagrams are shown in Fig. 19 (site A) and 20 (site B).

In order to represent the evolution of the mean critical angle throughout the quarry's lifetime a specific diagram is plotted, as shown in Fig. 19-22 for site A and site $\mathrm{B}$, respectively. 
In order to validate the results, data obtained by means of the exposed procedure are put in comparison with those measured in reality. Each site was characterized according with the following parameters: Extent of the exposed quarried area and extent of the zone of visibility. The comparison was carried out by concentrating on the actual configuration of the extraction site. Starting from the initial configuration of the deposit and imposing the annual production recorded in the previous years and then plotting the exploitation method really performed, the evolution of the various phases of quarrying was simulated for annual time intervals until the contemporary layout. The comparison between the two sets of values is summarized in the following table and it refers on single bench exploitation method for both sites.

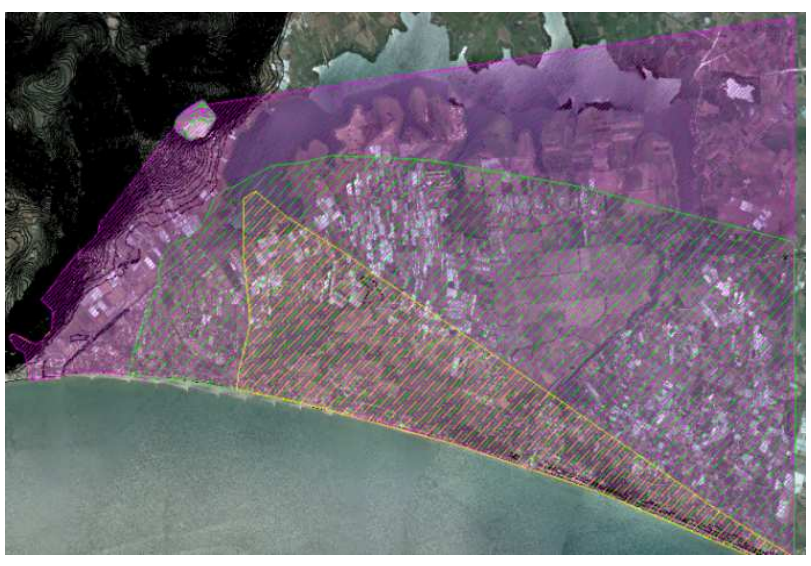

Fig. 18. Site B, single bench with reclamation visibility chart

\section{IVD Site A}

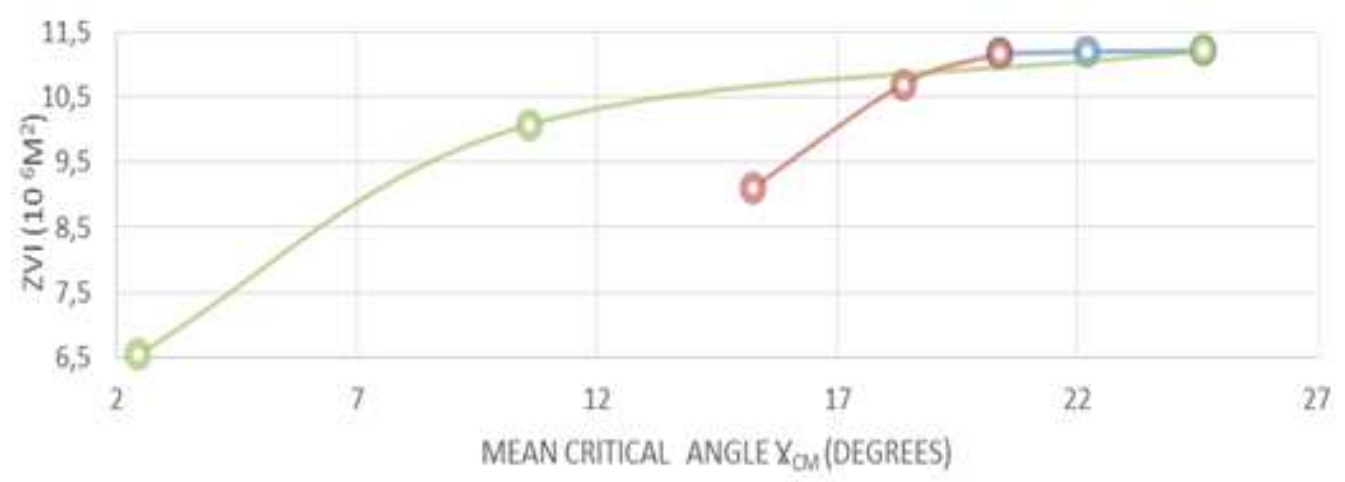

- - -Single bench $\rightarrow$-vertical slices -0 -single bench with restoration

Fig. 19. Site A IVD

\section{IVD Site B}

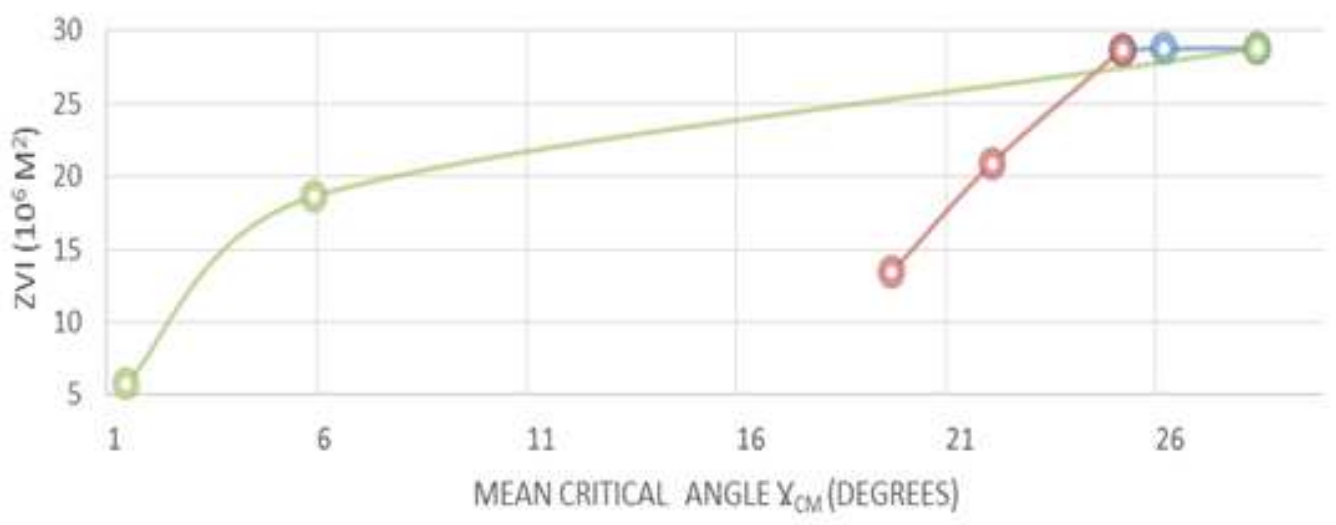

-0-Single bench $\quad-0$-Vertical slices $\quad-0$-single bench with restoration

Fig. 20. IVD Site B 


\section{Site A}

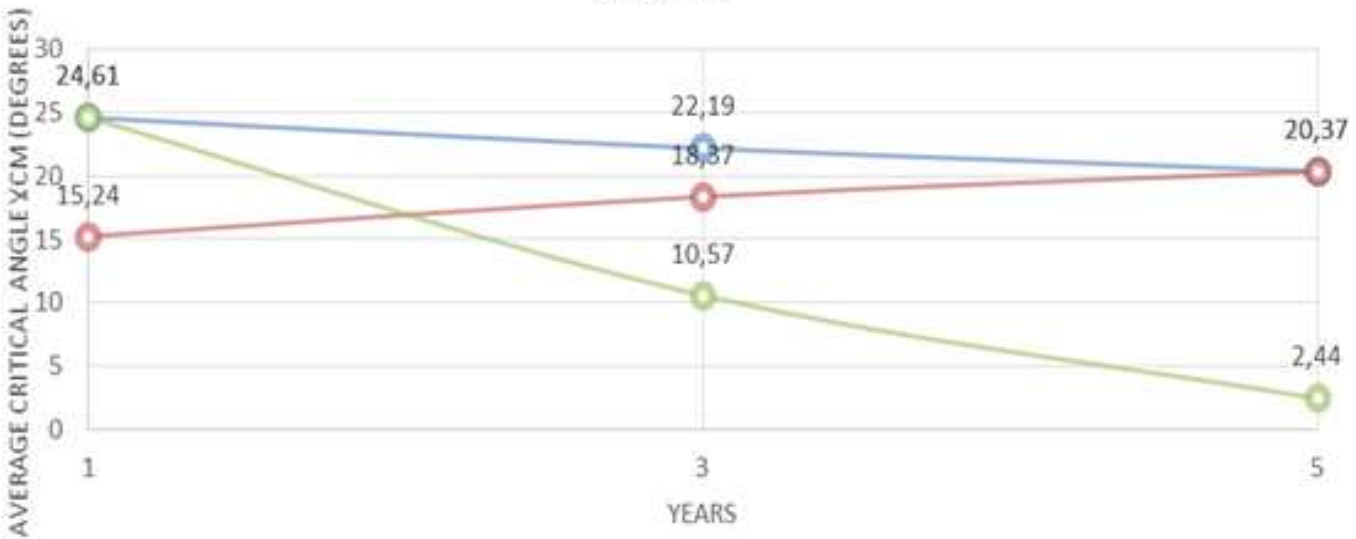

\section{-Single bench $\quad-0$-Vertical slices $\rightarrow-$ Single bench with restoration}

Fig. 21. Mean critical angle throughout years of extraction for site A

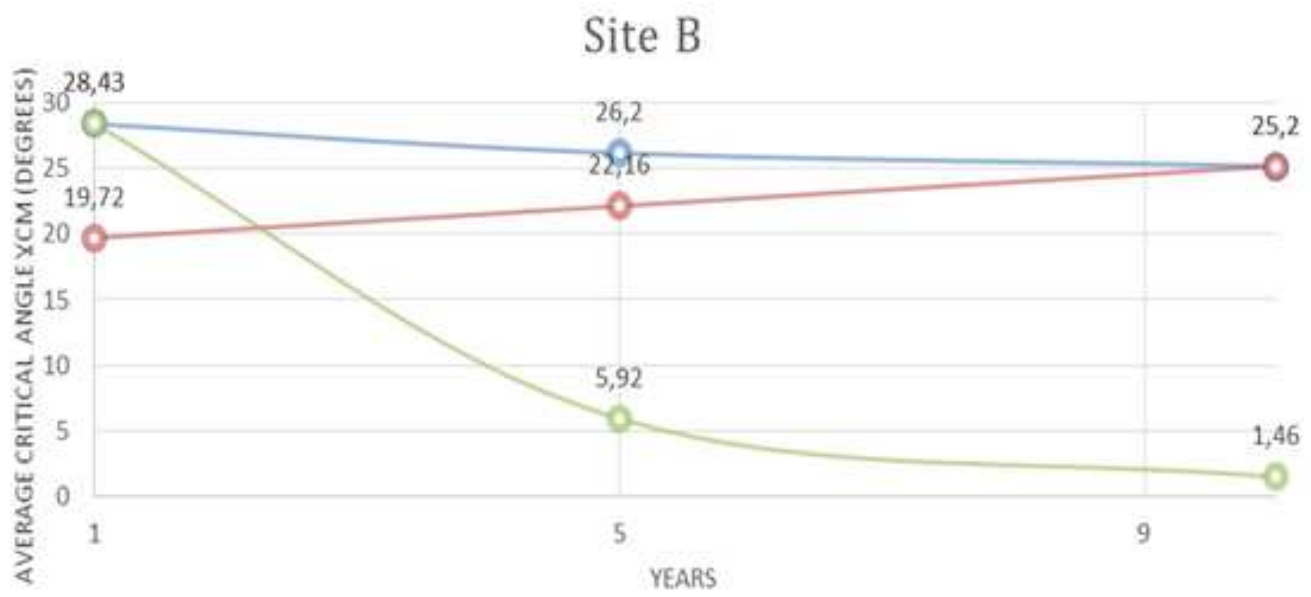

- Single bench

-Single bench with restoration

Fig. 22. Mean critical angle throughout years of extraction for site B

Table 3. Site A, the extent of the ZVI

\begin{tabular}{llll}
\hline & Zone of visual influence $\left[10^{3} \mathrm{~m}^{2}\right]$ & & \\
Exploitation method & Year 1 & Year 3 & $11.183,832$ \\
\hline Single bench & $11.223,926$ & $11.207,563$ & $11.183,832$ \\
Vertical slices & $9.110,041$ & $10.710,309$ & $6.542,890$ \\
Single bench with restoration & $11.223,92$ & $10.084,75$ & \\
\hline & & & \\
Table 4. Site B, the extent of the ZVI & & & \\
\hline & Zone of visual influence $\left[10^{3} \mathrm{~m}^{2}\right]$ & & Year 10 \\
\hline Exploitation method & - Year 1 & $28.752,977$ & $28.717,324$ \\
Single bench & $28.809,357$ & $20.941,363$ & $28.717,324$ \\
Vertical slices & $13.461,186$ & $18.625,66$ & $5.813,950$ \\
Single bench with restoration & $28.809,35$ &
\end{tabular}


Table 5. Site A: Average critical angle

\begin{tabular}{|c|c|c|c|}
\hline \multirow[b]{2}{*}{ Exploitation method } & \multicolumn{3}{|c|}{ Mean critical angle [degrees] } \\
\hline & Year 1 & Year 3 & Year 5 \\
\hline Single bench & 24,61 & 22,19 & 20,37 \\
\hline Vertical slices & 15,24 & 18,37 & 20,37 \\
\hline Single bench with restoration & 24,61 & 10,57 & 2,44 \\
\hline
\end{tabular}

Table 6. Site B: Average critical angle

\begin{tabular}{|c|c|c|c|}
\hline \multirow[b]{2}{*}{ Exploitation method } & \multicolumn{3}{|c|}{ Mean critical angle [degrees] } \\
\hline & Year 1 & Year 5 & Year 10 \\
\hline Single bench & 28,43 & 26,20 & 25,20 \\
\hline Vertical slices & 19,72 & 22,16 & 25,20 \\
\hline Single bench with restoration & 28,43 & 5,92 & 1,46 \\
\hline
\end{tabular}

Table 7. Comparison between forecasted and measured data for both sites

\begin{tabular}{|c|c|c|c|c|}
\hline \multirow[b]{2}{*}{ Site } & \multicolumn{2}{|c|}{ Exposed quarry area $\left[\mathrm{m}^{2}\right]$} & \multicolumn{2}{|c|}{ Zone of Visibility $\left[\mathrm{m}^{2}\right]$} \\
\hline & Measured & Forecasted & Measured & Forecasted \\
\hline$\overline{\mathrm{A}}$ & $32.055,00$ & $31.177,00$ & $11.018,00$ & $11.204,533$ \\
\hline B & $54.632,00$ & $52.927,38$ & $28.132,00$ & $28.654,76$ \\
\hline
\end{tabular}

\section{Discussion}

The analysis of the polar diagrams of the Exposed Quarry Area (EQA) suggests some considerations. First of all, as expected, the extent of the EQA increases throughout the quarry's lifetime both for the single bench method and vertical slices method. Moreover, the extent of the EQA reaches the same value when the all of the available deposit has been quarried. This feature implies that different exploitation methods applied to the same site result in the same exposed quarried area when the deposit has been completely removed and thus the final layout of the site is the same. On the other hand, there is a considerable difference noted when comparing the temporary evolution of the EQA. In fact the EQA generated by the single bench method grows faster than that with vertical slices and consequently the altered surface of the former is more visible from the initial stage of the quarry whereas the latter method allows a more gradual increase in visual impact (Table 1 and 2). This outcome, however, should not be generalized, as visual impact depends on the topographical characteristics of the site and the surrounding area. On the other hand the effect of the reclamation process may be appreciated with the method of single bench with reclamation which guarantees a reduction in the EQA. Although at the initial stage of the quarry, the EQA value of single bench with reclamation is the same as that of single bench, it tends to decrease during the extracting phase and becomes smaller than that of vertical slices. This tendency is observed in both case studies and happens during the second year for site A and during the fourth year for site B. As shown in Fig. 1 and 2, the quarries in these case studies are hill-side quarries rather than valley quarries. This factor influences the angle of vision and the outcomes of this particular study refer to the case in which the observer is at a lower or equal altitude to that of the quarry. In this case, since the angle of vision is positive, the horizontal surface of the quarry site remains hidden from view and the only exposed surface is the vertical face (projected onto a plane which is orthogonal to the direction of observation). Should this hypothesis change (i.e., when the observer is at a greater altitude than the quarry floor such as is frequently the case with a valley quarry), the horizontal surface would become visible to the observer and further study would be required (Alfaro Degan et al., 2015). A final observation concerning the EQA regards the characteristics of polar diagrams. Since the EQA depends on the azimuthal angle of view, once the maximum value of the EQA has been determined (i.e., determining the extent of the projection of the EQA itself onto a plane orthogonal to the view direction), the value of the EQA with reference to any other direction is calculated from the cosine of the angle the view direction forms with that of the maximum EQA. This feature is confirmed by the shape of the curves obtained experimentally, which are circumferences in both cases. Moreover, the analysis of the visibility charts shows some interesting outcomes relating to the Zones of Visual Influence (ZVI). With regard to both sites, as reported in the last column (rows 1 and 2) of Table 3 and 4 , the final extent of the ZVI of the single bench and vertical slices methods is the same. These two methods, 
which generate the same final layout (Table 1 and 2), also produce the same effect on the surroundings when the deposit has been fully removed. The extent of the ZVI also shows a temporary evolution depending on the method adopted. With regard to the single bench method, the ZVI reaches its maximum value at the end of the first year of exploitation and thereafter the value remains fairly constant and even decreases, albeit minimally. In contrast, with the vertical slices method the ZVI indicates a more steady growth throughout the years which vary, from one site to another, depending on the topography of the surrounding land. With regard to the method of single bench with reclamation, the ZVI area shows a reduction towards the end of the lifetime of the quarry while the extractive activities are still under way, confirming the effectiveness of this excavation method. Although the alteration to the landscape from this method is initially the same as that from the single bench method, it produces by far the least ZVI over time of the three methods in both sites. The values of the critical angle throughout the lifetime of each quarry are reported for the three different methods for sites A and B in Table 5 and 6 , respectively. In both sites the critical angle tends to increase when the vertical slices method is used while it decreases when the single bench method is used whether or not reclamation is employed. However, this reduction is much more pronounced when reclamation is carried out. Moreover, the Inter visibility Diagram (IVD) graph shows that in both sites, the ZVI increases (or at least remains constant) if the critical angle increases. On comparing the two diagrams it should be noted that at the beginning of the quarry's lifetime the vertical slices method generates the smallest critical angle, whereas since the effects of reclamation tend to be efficient, the single bench with reclamation method performs best at reducing the critical angle itself.

As shown in Table 7, the comparison of measured data and estimated ones allows a number of considerations to be made. Firstly, it should be noticed that in the case of the exposed quarried area, the application of the method returns, for both sites, a generalized underestimation of the values. This is because the extent of EQA was calculated from the project specifications of the deposit without carrying out any sample or measurement in the field. Such an omission did not allow to take into considerations local variations in the realization of single benches (i.e., presence of fractures or overburden). This feature, however, does not affect the quality of the estimate as, in both cases, the value of measured data is higher than that of the forecasted one by a factor lower than $5 \%$.

The second important outcome is referred to the extent of the zone of visibility. In both cases this value is overestimated by the method. A possible factor contributing to this overestimation when assessing the extent of ZVI by means of a design method may be linked to the selected scale of analysis. The ZVI is in fact obtained starting from the topographic map of the area of interest. The accuracy of the estimate also depends upon the details of the selected map. The presence of screens, trees or any other obstacle between the quarry and the observer may affect the assessment of the value. Nevertheless the adoption of GIS map may be suggested to prevent these fluctuations to be perceived.

\section{Conclusion}

This study identifies some parameters to quantitatively assess the visual impact of quarrying. It also presents the results obtained on applying the proposed procedure to two case studies which were chosen from the same geographical area. On the basis of the obtained results, the procedure, consisting in a multi-stage method, seems to be a useful tool. The first stage consists in a quantitative analysis of the exposed quarried surface as viewed by an observer. The Exposed Quarry Area (EQA) provides a rough assessment of the differences in impact due to the adoption of various quarrying methods. Parameters set at this stage in order to draw polar diagrams allow the type extraction method to be taken into account (whereas the characteristics of the surrounding topography are not considered until a later stage). The second stage defines the Zone of Visual Influence (ZVI) parameter which examines the extent to which the quarry plant affects the surrounding landscape. The ZVI parameter allows the visual intrusion generated by the plant to be quantified and its area plotted on a map. This phase determines whether or not given receptors will be affected by the view of the quarry. The last step merges the quarry design parameters with the topographical parameters by determining the mean critical angle. The mean critical angle may be assessed for any point in time of the quarry and is the maximum angle beyond which the EQA is no longer visible to an observer. Therefore, once the contour map of the area is available and a certain view has been selected, the mean critical angle may be used as input data during the decision making stages of quarry planning. This may be used to decide upon the exploitation plan in terms of spatial development of the excavations in general and in particular when choosing the ratio between the height and width of the benches. In conclusion, although the choice of exploitation method is connected with many factors involving geostructural features (such as the type of rock, the structure of the deposit, the stability of the quarry face) or economic aspects (especially with regard to the quarry production rate), this study highlights a simple way to include parameters pertaining to landscape alteration as design constraints to minimize visual intrusion. 


\section{Funding Information}

This research was developed by the Scientific Research Fund of Roma Tre University, Engineering Dept.

\section{Author's Contributions}

Dario Lippiello: Designed the research plan, participated in all experiments, coordinated the data analysis and contributed to the writing of the paper.

Guido Alfaro Degan: Organized the study, participated in all experiments and contributed to the writing of the paper.

Marco De Agostini: Participated in all experiments and in the data analysis also contributing to the writing of the paper.

Mario Pinzari: Critically reviewed the manuscript.

\section{Ethics}

All of the other authors have read and approved the manuscript and there are no ethical issues involved.

\section{References}

Alfaro Degan, G., D. Lippiello, L. Picciolo and M. Pinzari, 2014. Visual impact from quarrying activities: A case study for planning the residential development of surrounding areas. WIT Trans. Ecol. Environ., 181: 125-135. DOI: 10.2495/EID140111

Alfaro Degan, G., D. Lippiello and M. Pinzari, 2015. Landscape changes due to quarrying activities as a project parameter for urban planning. Int. J. Sustainable Develop. Plann., 10: 843-862. DOI: $10.2495 /$ SDP-V10-N6-843-862

Bishop, I.D., 2003. Assessment of Visual Qualities, Impacts and Behaviours, in the Landscape, by Using Measures of Visibility. Environ. Plan. B: Plan. Design, 30: 677-688. DOI: 10.1068/b12956

Briggs, D.J. and J. France, 1980. Landscape evaluation: A comparative study. J. Environ. Manage., 10: 263-275.

Cardu, M., M. Fornaro and I. Sacerdote, 2001. Economic-environmental description of the exploitation method by horizontal slices and ore pass in Italian surface crushed stone quarries. Proceedings of the 17th International Mining Congress and Exhibition of Turkey, (CET' 01), pp: 561-566.

Gasparovic, S., A. Mrda and L. Petrovic, 2009. Landscape models of reclamation and conversion of quarries recovering landscapes. Prostor, 17: 372-385.
Hernandez, J., L. Garcia and F. Ayuga, 2004. Assessment of the visual impact made on the landscape by new buildings: A methodology for site selection. Landscape Urban Plann., 68: 15-28. DOI: 10.1016/S0169-2046(03)00116-6

Jordan, M.M., 2007. Geological and environmental implications in the reclamation of limestone quarries in Sierra de Callosa (Alicante, Spain). Environ. Earth Sci., 59: 687-694. DOI: 10.1007/s12665-009-0065-x

Kumar Dey, P. and E. Ramcharan, 2008. Analytic hierarchy process helps select site for limestone quarry expansion in Barbados. J. Environ. Manage., 88: 1384-1395. DOI: 10.1016/j.jenvman.2007.07.011

Massacci, G. and V. Dentoni, 2007. Visibility of surface mining and impact perception. Int. J. Min. Reclamat. Environ., 21: 6-13. DOI: $10.1080 / 17457300600906289$

Menegaki, M., I. Koutiva and D. Kaliampakos, 2015. Assessing the chromatic contrast in open surface excavations: A comparative study between subjective and quantitative approaches. Int. J. Min. Reclamat. Environ., 29: 112-124. DOI: 0.1080/17480930.2013.866791

Menegaki, M.E. and D.C. Kaliampakos, 2006. Landscape analysis as a tool for surface mining design. Environ. Plann. B: Plann. Design, 33: 185-196.

DOI: $10.1068 / \mathrm{b} 31005$

Mouflis, G.D., Z.D. Ioannis, S. Iliadou and G.H. Mitri, 2008. Assessment of the visual impact of marble quarry expansion (1984-2000) on the landscape of Thasos island, NE Greece. Landscape Urban Plann., 86: 92-102. DOI: 10.1016/j.landurbplan.2007.12.009

Panagopoulos, T., R. Matias and B.R. Ramos, 2007. Visual impact and reclamation of limestone quarries in Algarve Portugal. Proceedings of the 24th National Meetings of the American Society of Mining and Reclamation, Sept. 1-3, Rhodes island, Greece, pp: 176-182. DOI: 10.21000/JASMR07010611

Pinto, V., S. Font, M. Salgot, J. Tapias and T. Mañá, 2002. Image analysis applied to quantitative evaluation of chromatic impact generated by openpit quarries and mines. Environ. Geol., 41: 495-503. DOI: $10.1007 / \mathrm{s} 002540100259$

Pinzari, M. and F. Valente, 1992. Quarry landscape Analysis. Quarry Construct., 8: 17-26.

Shafer, E.L., 1969. Perception of natural environments. Environ. Behaviour, 1: 71-82. DOI: $10.1177 / 001391656900100105$ 